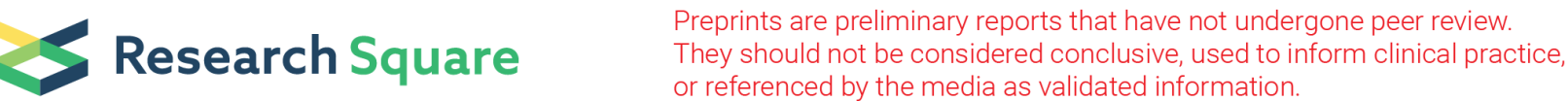

\section{F-Alfatide II for the evaluation of axillary lymph nodes in breast cancer patients: comparison with 18F-FDG}

\section{Jiang Wu}

Nanjing Jinling Hospital: East Region Military Command General Hospital

Jihong Tian

Nanjing Medical University

Yiyan Zhang

Nanjing University of Chinese Medicine

\section{Hengshan Ji}

Nanjing Jinling Hospital: East Region Military Command General Hospital

\section{Jingjing Sun}

Nanjing Jinling Hospital: East Region Military Command General Hospital

\section{Xingang Wang}

Nanjing Jinling Hospital: East Region Military Command General Hospital

Chuanjin Sun

Nanjing Jinling Hospital: East Region Military Command General Hospital

\section{Zhaogang Teng}

Nanjing University of Posts and Telecommunications

Hong Zhu

Nanjing Jinling Hospital: East Region Military Command General Hospital

Xiaoyuan Chen ( $\nabla$ chen.shawn@nus.edu.sg)

National University of Singapore - Kent Ridge Campus: National University of Singapore https://orcid.org/0000-0002-9622-0870

\section{Research Article}

Keywords: 18F-Alfatide II, integrin av $\beta 3$, positron emission tomography, breast cancer, axillary lymph node

Posted Date: February 15th, 2021

DOI: https://doi.org/10.21203/rs.3.rs-166779/v1

License: (c) (i) This work is licensed under a Creative Commons Attribution 4.0 International License. Read Full License 
Version of Record: A version of this preprint was published at European Journal of Nuclear Medicine and Molecular Imaging on February 9th, 2022. See the published version at https://doi.org/10.1007/s00259021-05333-z. 


\section{Abstract}

${ }^{18} \mathrm{~F}$-Alfatide II has been translated into clinical use and been proven to have good performance in identifying breast cancer. In this study, we investigated ${ }^{18} \mathrm{~F}$-Alfatide II for evaluation of axillary lymph nodes (ALN) in breast cancer patients and compared the performances with ${ }^{18} \mathrm{~F}$-FDG.

Methods: A total of 44 female patients with clinically suspected breast cancer were enrolled and underwent ${ }^{18} \mathrm{~F}$-Alfatide II and ${ }^{18} \mathrm{~F}$-FDG PET/CT within a week. Tracer uptakes in ALN were evaluated by visual analysis, semiquantitative analysis with maximum standardized uptake value $\left(S U V_{\text {max }}\right)$, mean

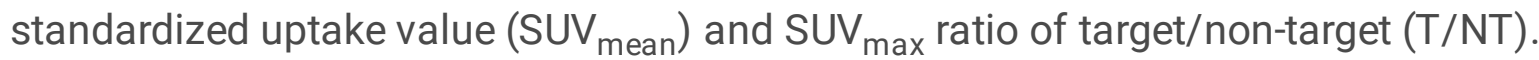

Results: Among 44 patients, 37 patients were pathologically diagnosed with breast cancer with metastatic (17 cases) or non-metastatic (20 cases) ALN. The sensitivity, specificity, accuracy, positive predictive value (PPV) and negative predictive value (NPV) of visual analysis were $70.59 \%, 90.00 \%$, $81.08 \%, 85.71 \%$ and $78.26 \%$ for ${ }^{18} \mathrm{~F}$-Alfatide $I I, 64.71 \%, 90.00 \%, 78.38 \%, 84.62 \%$ and $75.00 \%$ for ${ }^{18} \mathrm{~F}$-FDG, respectively. By combining ${ }^{18} \mathrm{~F}$-Alfatide II and ${ }^{18} \mathrm{~F}$-FDG, the sensitivity significantly increased to $82.35 \%$, the specificity was $85.00 \%$, the accuracy increased to $83.78 \%$, the PPV was $82.35 \%$ and the NPV significantly increased to $85.00 \%$. Three cases of luminal B subtype were false negative for both ${ }^{18} \mathrm{~F}-$ Alfatide II and ${ }^{18} \mathrm{~F}$-FDG. The other 2 false negative cases of ${ }^{18} \mathrm{~F}$-Alfatide II were triple-negative subtype and 3 false negative cases of ${ }^{18} \mathrm{~F}-\mathrm{FDG}$ were luminal B subtype too. The AUCs of three semi-quantitative parameters $\left(S_{\text {max }}, S U V_{\text {mean }}, T / N T\right)$ for ${ }^{18} \mathrm{~F}$-Alfatide II were between 0.8 and 0.9 , whereas those for ${ }^{18} \mathrm{~F}$ FDG were more than 0.9. ${ }^{18} \mathrm{~F}$-Alfatide II T/NT had the highest Youden index $(76.5 \%)$, specificity $(100 \%)$, accuracy $(89.19 \%)$ and PPV $(100 \%)$ among these semi-quantitative parameters. ${ }^{18} \mathrm{~F}$-Alfatide II uptake as well as ${ }^{18} \mathrm{~F}$-FDG uptake in metastatic axillary lymph nodes (MALN) was significantly higher than that in benign axillary lymph nodes (BALN). Both ${ }^{18} \mathrm{~F}$-Alfatide II and ${ }^{18} \mathrm{~F}$-FDG did not show difference in primary tumor uptake irrespective of ALN status.

Conclusion: ${ }^{18} \mathrm{~F}$-Alfatide II can be used in breast cancer patients to detect metastatic ALN, however, like ${ }^{18} \mathrm{~F}$-FDG, with high specificity but relatively low sensitivity. The combination of ${ }^{18} \mathrm{~F}$-Alfatide II and ${ }^{18} \mathrm{~F}$-FDG can significantly improve sensitivity and NPV. ${ }^{18} \mathrm{~F}$-Alfatide II T/NT may serve as the most important semiquantitative parameter to evaluate ALN.

\section{Introduction}

Axillary lymph nodes (ALN) are the most common spread site of breast cancer $(1,2)$. The status of ALN determines the staging, treatment strategy and prognosis for breast cancer patients (3). ALN involvement often means the more advanced stage, the greater extent of surgery, the need for systemic therapy and the lower survival rate. ALN metastasis is also the most important predictor of overall recurrence and survival (4). Therefore, the identification of ALN metastasis is crucial for patients with breast cancer. The gold standard for detecting ALN involvement is ALN dissection, which however can bring many adverse 
complications such as arm edema, pain, neuropathy, and so on (5). Although minimally invasive methods including sentinel lymph node biopsy, core needle biopsy and fine needle aspiration cytology lead to fewer complications than ALN dissection, they have the risk of implantation metastasis (6). For these reasons, a non-invasive method of evaluating ALN is essential.

X-ray mammography, ultrasound, CT and magnetic resonance imaging (MRI) are traditional imaging methods, which can non-invasively evaluate ALN. However, the evaluation using these imaging methods is based on anatomical abnormalities and morphological changes, which often fail to reflect the status of ALN accurately (1). As a molecular imaging method, positron emission tomography (PET) has its superiority to traditional imaging methods in the clinic. It has been widely accepted for the diagnosis, staging, treatment evaluation and recurrence monitoring in cancer patients. As the most frequently used PET tracer, ${ }^{18} \mathrm{~F}-\mathrm{FDG}$ can provide glucose metabolism information in tissues. Many ${ }^{18} \mathrm{~F}$-FDG PET or $\mathrm{PET} / \mathrm{CT}$ studies have also shown promising results for ALN evaluation. It has been reported that the specificity of ${ }^{18} \mathrm{~F}-\mathrm{FDG}$ for detecting metastatic ALN is up to $90-100 \%$, but the sensitivity is relatively low to $50-80 \%$ (7-9). Accordingly, ${ }^{18} \mathrm{~F}-\mathrm{FDG}$ PET or PET/CT may underestimate the number of metastatic ALN. Therefore, more accurate ALN assessment entails some emerging PET tracers.

${ }^{18} \mathrm{~F}$-Alfatide II is an arginine-glycine-aspartate (RGD) based PET tracer targeting integrin $\mathrm{a}_{\mathrm{v}} \beta_{3}$, which is highly expressed on activated endothelial cells of tumor neovasculature $(10,11)$. Some studies have shown the usefulness of ${ }^{18} \mathrm{~F}$-Alfatide II for tumor detection, therapy response prediction and prognosis evaluation (12-20). One of our previous clinical studies also demonstrated that ${ }^{18} \mathrm{~F}$-Alfatide II has good performance in identifying breast cancer. Particularly, ${ }^{18} \mathrm{~F}$-Alfatide II may be superior to ${ }^{18} \mathrm{~F}$-FDG in detecting breast cancer with strongly positive ER but negative HER-2 expression (21). Moreover, another clinical study also indicated that this tracer is valuable in the diagnosis of metastatic lymph nodes for non-small cell lung cancer patients (22). However, there is yet no report concerning ${ }^{18} \mathrm{~F}$-Alfatide II in detecting metastatic ALN of breast cancer.

In this work, ${ }^{18} \mathrm{~F}$-Alfatide II was further investigated in breast cancer patients for assessing ALN on the basis of our previous study (21). We obtained diagnostic parameters of ${ }^{18} \mathrm{~F}$-Alfatide II for identifying ALN metastasis using visual and semi-quantitative methods and assessed its diagnostic performance as compared to ${ }^{18} \mathrm{~F}$-FDG. We also compared diagnostic parameters of ${ }^{18} \mathrm{~F}$-Alfatide II and ${ }^{18} \mathrm{~F}$-FDG among different groups of ALN.

\section{Materials And Methods}

\section{Patients}

This study was approved by the ethics committee of Jinling Hospital (Approval No. 2015NZYW-007) and registered at ClinicalTrials.gov (NCT02582801). The including criteria include: (1) clinically suspected primary breast cancer according to conventional imaging, (2) no prior treatment for breast lesions, (3) 18 
$y<$ age $<70 y$. The excluding criteria include: (1) pregnancy or lactation period, (2) some accompanied serious diseases (e.g., impaired liver or renal function, active tuberculosis, another malignant tumor). Forty-four female patients (age range, 28-66 y, mean age, $50.73 \pm 8.01 \mathrm{y}$ ) were included in this study and written informed consent was obtained from each patient.

\section{PET/CT Acquisition}

${ }^{18} \mathrm{~F}$-Alfatide II was synthesized according to the previously described method (23). PET/CT scans with ${ }^{18} \mathrm{~F}$-Alfatide II and ${ }^{18} \mathrm{~F}$-FDG were performed with an interval of at least one day and completed within one week. No specific patient preparation was requested before ${ }^{18} \mathrm{~F}$-Alfatide II injection, but fasting for at least $6 \mathrm{~h}$ was requested before ${ }^{18} \mathrm{~F}-\mathrm{FDG}$ injection. The injected activity was $306 \pm 80 \mathrm{MBq}$ (range 155-503 $\mathrm{MBq}$ ) for ${ }^{18} \mathrm{~F}$-Alfatide II and approximately $3.7 \mathrm{MBq} / \mathrm{kg}$ body weight for ${ }^{18} \mathrm{~F}$-FDG. In addition, blood glucose level below $140 \mathrm{mg} / \mathrm{dL}$ needs to be confirmed before ${ }^{18} \mathrm{~F}-\mathrm{FDG}$ injection. After a rest for $60 \mathrm{~min}$ after injection, patients underwent the scans using an integrated PET/CT system (Biography 16, Siemens Healthcare). CT acquisition was initially performed with $120 \mathrm{kV}, 140 \mathrm{~mA}$, and a slice thickness of $5 \mathrm{~mm}$. Subsequently, PET acquisition was performed with $3 \mathrm{~min}$ for each bed position. The CT data were used for PET attenuation correction and PET images were reconstructed using an iterative algorithm.

\section{Image Analysis}

PET, CT, and fused images were displayed on a Siemens/Syngo user interface. The images were interpreted by a consensus of two experienced nuclear medicine physicians, who were blinded to the histological diagnosis and other imaging results. It was defined as visually positive that the radioactive uptake of the ALN was higher than that of the surrounding normal tissue. The maximum standardized uptake value $\left(S U V_{\text {max }}\right)$ and the mean standardized uptake value $\left(S U V_{\text {mean }}\right)$ were semi-quantitatively measured by drawing regions of interest over the ALN. The SUV max ratio of the ALN on the same side of the breast lesion to the node on the opposite side was calculated as T/NT (target/non-target).

\section{Statistical Analysis}

Statistical analysis was performed using SPSS software (version 17.0, SPSS, Inc., Chicago, IL, USA). All semi-quantitative data were expressed as the mean \pm standard deviation. Differences both in independent samples and in paired samples were analyzed by $t$-test. The sensitivity, specificity, accuracy, PPV and NPV were calculated for each diagnostic parameter. Receiver operating characteristic (ROC) curve analysis was performed to evaluate the diagnostic performance. The area under the curve (AUC) and the cutoff value were further determined at the point with the highest Youden index. A $P$ value less than 0.05 was considered statistically significant.

\section{Results}

\section{Pathological Findings}


All 44 patients received biopsy or surgery on breast lesion after two PET/CT scans. Among them, 39 patients were pathologically diagnosed with breast cancer and the other 5 were diagnosed with benign breast disease. In 39 patients with breast cancer, 2 patients did not undergo biopsy or surgery on ALN thus unclear about the metastasis status. The pathological results of ALN were obtained in the remaining 37 breast cancer patients, out of which 17 cases were confirmed with ALN metastasis and 20 cases were without. Characteristics of these 37 patients are presented in Table 1. Based on immunohistochemistry and fluorescence in situ hybridization (FISH), the invasive carcinomas in 33 patients (excluding 4 patients with ductal carcinoma in situ) were further divided into four molecular subtypes. ALN metastasis occurred in 2 cases of luminal A subtype (2/5), 8 cases of luminal B subtype (8/14), 3 cases of HER-2 overexpressing subtype (3/4) and 4 cases of triple-negative subtype (4/10).

Table 1

Characteristics of Breast Cancer Patients for Assessing ALN

\begin{tabular}{|l|}
\hline Variable Breast Cancer (n) \\
Median Age (years, range) 53 (28-66) \\
Histology 37 \\
Ductal Carcinoma in situ 4 \\
Invasive Carcinoma 33 \\
Molecular Subtype 33 \\
Luminal A 5 \\
Luminal B 14 \\
HER-2 overexpressing 4 \\
Triple-negative 10 \\
Axillary Lymph Node 37 \\
Metastasis 17 \\
No Metastasis 20 \\
\hline
\end{tabular}

\section{Visual Assessment Findings}

Among 17 cases with ALN metastasis, 12 cases were correctly recognized using ${ }^{18} \mathrm{~F}$-Alfatide II (Fig. 1) and 5 cases were false negative (Fig. 2). In comparison, 11 cases were correctly identified using ${ }^{18} \mathrm{~F}$-FDG (Fig. 1) and 6 cases were false negative (Fig. 2). By combining ${ }^{18}$ F-Alfatide II and ${ }^{18} \mathrm{~F}$-FDG, the number of false negative cases was three. Among 20 cases without ALN metastasis, 18 cases were true negative, and 2 cases were false positive using ${ }^{18} \mathrm{~F}$-Alfatide II. Likewise, the number of true negative cases was 18 and that of false positive cases was 2 for ${ }^{18} \mathrm{~F}$-FDG. There was one false positive case for both ${ }^{18} \mathrm{~F}$ Alfatide II and ${ }^{18} \mathrm{~F}$-FDG. By combining the two tracers, the number of false positive cases was three. The 
corresponding sensitivity, specificity, accuracy, positive predictive value (PPV) and negative predictive value (NPV) are shown in Table 2. Both ${ }^{18} \mathrm{~F}$-Alfatide II and ${ }^{18} \mathrm{~F}$-FDG had the same high specificity (90.00\%) and relatively low sensitivity $(70.59 \%$ vs. $64.71 \%)$. They both had similar accuracy $(81.08 \%$ vs. $78.38 \%)$, PPV (85.71\% vs. $84.62 \%)$ and NPV (78.26\% vs. 75.00\%). Moreover, the combined sensitivity and NPV significantly increased to $82.35 \%$ and $85.00 \%$, while the combined accuracy increased to $83.78 \%$.

Table 2

Visual Analysis of ${ }^{18} \mathrm{~F}$-Alfatide II and ${ }^{18} \mathrm{~F}$-FDG in Identifying ALN Metastasis

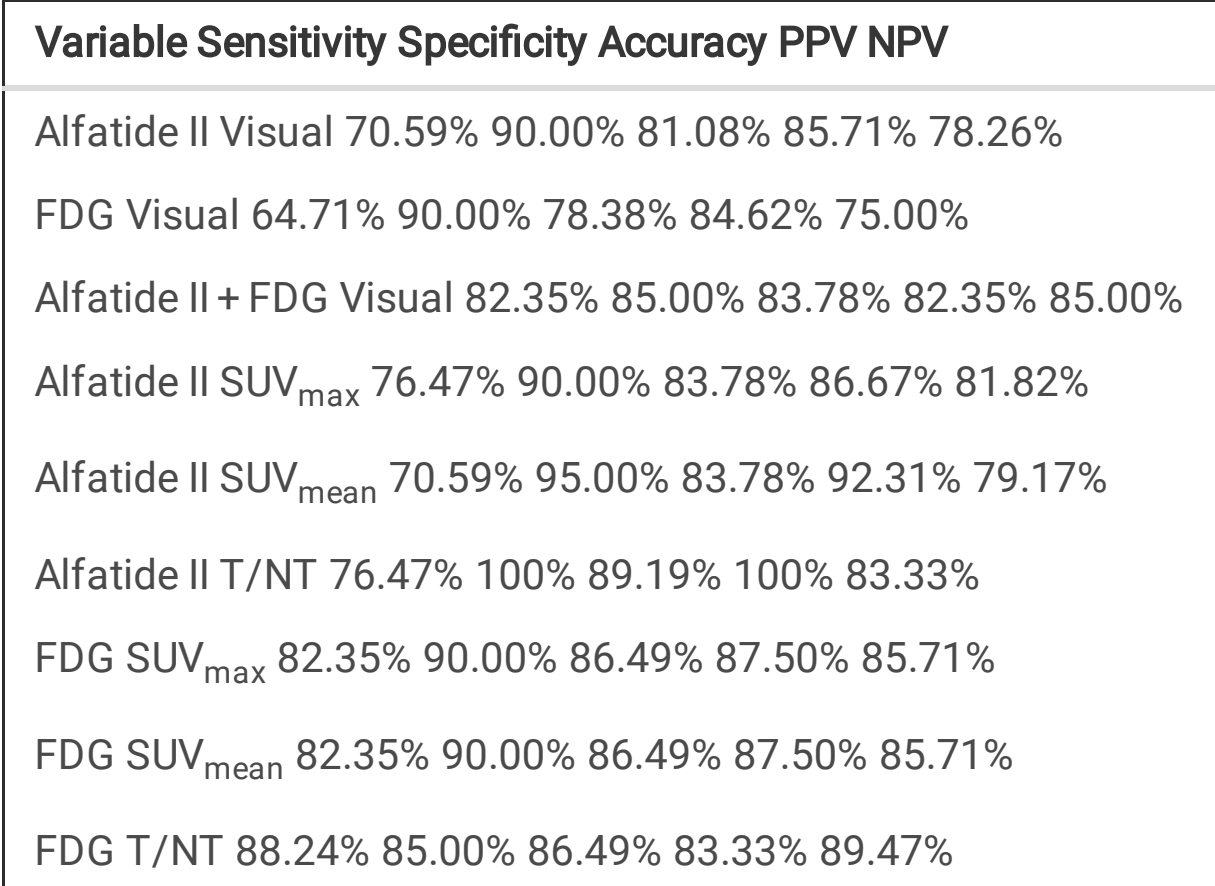

There were three false negative cases for both ${ }^{18} \mathrm{~F}$-Alfatide II and ${ }^{18} \mathrm{~F}$-FDG. All the 3 cases belonged to luminal B subtype (Fig. 2). The other 2 false negative cases for ${ }^{18} \mathrm{~F}$-Alfatide II were triple-negative subtype (Fig. 3). The remaining 3 false negative cases for ${ }^{18} \mathrm{~F}-\mathrm{FDG}$ were all luminal B subtype (Fig. 4). One case of ductal carcinoma in situ was false positive for both ${ }^{18} \mathrm{~F}$-Alfatide II and ${ }^{18} \mathrm{~F}$-FDG (Fig. 5). The other false positive case for ${ }^{18} \mathrm{~F}$-Alfatide II was luminal B subtype and that for ${ }^{18} \mathrm{~F}$-FDG was HER-2 overexpressing subtype.

\section{Semi-quantitative Assessment Findings}

ROC curves of various semi-quantitative parameters are shown in Fig. 6 . For both ${ }^{18} \mathrm{~F}$-Alfatide II and ${ }^{18} \mathrm{~F}$ FDG, the curves are located at the upper left of the chance line, indicating their strong potential for identifying ALN metastasis in breast cancer patients. However, the AUCs of ${ }^{18} \mathrm{~F}$-Alfatide II are slightly less than those of ${ }^{18} \mathrm{~F}-\mathrm{FDG}$. The corresponding statistics of the ROC curves are presented in Table 3 . The AUCs of ${ }^{18} \mathrm{~F}$-Alfatide II are between 0.8 and 0.9 , while those of ${ }^{18} \mathrm{~F}$-FDG are all more than 0.9 . The maximum Youden indices are close among these semi-quantitative parameters. ${ }^{18} \mathrm{~F}$-Alfatide II T/NT has the highest Youden index (76.50\%), which indicates that the parameter has the highest diagnostic accuracy. Corresponding to the maximum Youden index, each semi-quantitative parameter has its cutoff 
value. Based on these cutoff values, the sensitivity, specificity, accuracy, PPV and NPV values are calculated in Table 2. Although the sensitivity of each ${ }^{18} \mathrm{~F}$-Alfatide II parameter is less than that of ${ }^{18} \mathrm{~F}$ FDG, the specificity of the former is superior or equal to that of the latter. In particular, ${ }^{18} \mathrm{~F}$-Alfatide II T/NT has the highest specificity (100\%), accuracy (89.19\%) and PPV (100\%) among all these parameters. The highest sensitivity (88.24\%) is for ${ }^{18}$ F-FDG T/NT, as well as the highest NPV (89.47\%).

Table 3

ROC Quantitative Analysis of ${ }^{18} \mathrm{~F}$-Alfatide II and

${ }^{18} \mathrm{~F}$-FDG in Identifying ALN Metastasis

\section{Parameter AUC $P$ Cutoff value Youden index}

Alfatide II SUV $\max 0.8120 .0011 .09566 .50 \%$

Alfatide II SUV mean $0.8040 .0020 .8165 .60 \%$

Alfatide II T/NT $0.8510 .0002 .576 .50 \%$

FDG SUV $\max 0.9240 .0001 .26572 .40 \%$

FDG SUV $V_{\text {mean }} 0.9210 .0000 .7972 .40 \%$

FDG T/NT $0.9220 .0002 .74573 .20 \%$

\section{Comparisons Among Different Groups of ALN}

The SUV max SUV mean and T/NT for ${ }^{18} \mathrm{~F}$-Alfatide II between metastatic axillary lymph node (MALN) group and non-metastatic axillary lymph node (i.e. benign axillary lymph node, BALN) group are compared in Table 4, as well as those for ${ }^{18} \mathrm{~F}-\mathrm{FDG}$. Using any of the three parameters, MALN had significantly higher ${ }^{18} \mathrm{~F}$-Alfatide II uptake than BALN $(P<0.05)$. The same result was obtained for ${ }^{18} \mathrm{~F}-\mathrm{FDG}$. The SUV $\mathrm{V}_{\text {max }}$ SUV $_{\text {mean }}$ and T/NT for the two tracers in the primary breast tumors with MALN and BALN are also compared in Table 5. No statistically significant differences between the two groups were found (all $P$ values were greater than 0.05 ). 
Table 4

Comparisons of ${ }^{18} \mathrm{~F}$-Alfatide II and ${ }^{18} \mathrm{~F}$-FDG Uptake between MALN and BALN

\begin{tabular}{|l|}
\hline Parameter MALN BALN $\boldsymbol{t} P$ \\
\hline Alfatide II SUV \\
Alfatide II SUV \\
Alfatide II T/NT $5.922 \pm 4.508 \pm 2.0340 .675 \pm 0.3794 .1750 .000$ \\
FDG SUV \\
FDax $4.173 \pm 3.7620 .833 \pm 0.7963 .8790 .000$ \\
FDG T/NT $12.348 \pm 12.3702 .079 \pm 1.5803 .6870 .001$ \\
\hline
\end{tabular}

Table 5

Comparisons of ${ }^{18} \mathrm{~F}$-Alfatide II and ${ }^{18} \mathrm{~F}$-FDG uptake between Primary Tumors with MALN and Primary Tumors with BALN.

\section{Parameter Primary Tumors with MALN Primary Tumors with BALN $t P$}

Alfatide II SUV $\max 4.205 \pm 2.1863 .563 \pm 1.5521 .0430 .304$

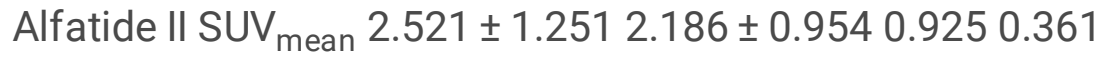

Alfatide II T/NT $6.330 \pm 4.0154 .719 \pm 2.1771 .5490 .130$

FDG SUV $\max 6.927 \pm 3.5857 .510 \pm 5.0020 .4010 .691$

FDG SUV ${ }_{\text {mean }} 4.230 \pm 2.1984 .686 \pm 3.2150 .4940 .625$

FDG T/NT 7.672 $\pm 5.09911 .385 \pm 15.8090 .9260 .361$

\section{Discussion}

${ }^{18} \mathrm{~F}$-Alfatide II is a PET probe to target integrin $\mathrm{a}_{\mathrm{v}} \beta_{3}$. Our previous study demonstrated the clinical usefulness of ${ }^{18} \mathrm{~F}$-Alfatide II in breast cancer, suggesting that it is comparable to ${ }^{18} \mathrm{~F}$-FDG for identification of breast cancer and can exert a complementary role to ${ }^{18} \mathrm{~F}-\mathrm{FDG}$ for some subtypes (21). When using ${ }^{18} \mathrm{~F}$-Alfatide II to detect lymph node metastases, Zhou's study (22) showed encouraging results with the sensitivity of $100 \%$, the specificity of $94.90 \%$ and the accuracy of $95.40 \%$ for non-small cell lung cancer patients.

In this study, visual assessment findings showed that the false negative rate of ${ }^{18} \mathrm{~F}$-Alfatide II (5/17) was similar to that of ${ }^{18} \mathrm{~F}$-FDG (6/17) for detecting metastatic ALN, whereas the false positive rate of ${ }^{18} \mathrm{~F}$ Alfatide II (2/20) was about the same as that of ${ }^{18} \mathrm{~F}-\mathrm{FDG}(2 / 20)$. The relatively low sensitivity $(64.71 \%)$ 
and high specificity $(90.00 \%)$ of ${ }^{18} \mathrm{~F}-\mathrm{FDG}$ in our study were consistent with the numbers reported in previous studies (24-31). Compared with ${ }^{18} \mathrm{~F}$-FDG, ${ }^{18} \mathrm{~F}$-Alfatide II had the same high specificity (90.00\%) and relatively higher sensitivity (70.59\%). Moreover, the accuracy, PPV and NPV of ${ }^{18} \mathrm{~F}$-Alfatide II were somewhat higher than those of ${ }^{18} \mathrm{~F}$-FDG. Although ${ }^{18} \mathrm{~F}$-Alfatide II was non-inferior to ${ }^{18} \mathrm{~F}$-FDG in detecting metastatic ALN, its sensitivity (70.59\%) was much lower than that reported by Zhou et al. in lung cancer patients, which was almost $100 \%$ (22). For both ${ }^{18} \mathrm{~F}$-Alfatide II and ${ }^{18} \mathrm{~F}$-FDG, the relatively high number of false negative cases is the main cause for the compromised sensitivity. However, combining ${ }^{18} \mathrm{~F}$-Alfatide II and ${ }^{18} \mathrm{~F}-\mathrm{FDG}$ together can significantly improve sensitivity and NPV, suggestive of their mutually complementary role.

For ${ }^{18} \mathrm{~F}-\mathrm{FDG}$, all 6 false negative cases were of luminal B subtype. Although there are still controversies on the correlation between hormonal receptor status, HER-2 overexpression and ${ }^{18} \mathrm{~F}$-FDG uptake $(32,33)$, ALN without ${ }^{18} \mathrm{~F}$-FDG foci should remain alerted for breast cancer patients of luminal B subtype. For ${ }^{18} \mathrm{~F}$ Alfatide II, three of the five false negative cases were also luminal B subtype, which may be attributed to the small sized ALN in the three patients. The other two false negative cases of ${ }^{18} \mathrm{~F}$-Alfatide II were triplenegative subtype. Our previous study also demonstrated that primary tumors of triple-negative subtype had the lowest ${ }^{18} \mathrm{~F}$-Alfatide II uptake compared with the other three subtypes (21). Thus ${ }^{18} \mathrm{~F}$-Alfatide II appears to bring about false negative results for assessing ALN of triple-negative subtype just like what we observed in assessing primary tumors. Based on our findings, ${ }^{18} \mathrm{~F}$-Alfatide II can play a complementary role to ${ }^{18} \mathrm{~F}$-FDG in assessing ALN of luminal B subtype, while ${ }^{18} \mathrm{~F}$-FDG can make up for the deficiency of ${ }^{18} \mathrm{~F}$-Alfatide II in evaluating ALN of triple-negative subtype.

According to semi-quantitative assessment findings in this study, three parameters (SUV $\mathrm{Sax}_{\text {, }} \mathrm{SUV}_{\text {mean }}$, $\mathrm{T} / \mathrm{NT}$ ) of ${ }^{18} \mathrm{~F}$-Alfatide II showed AUCs of more than 0.8 , indicating their strong potential for detecting metastatic ALN. As presented in Table 3, the optimal cutoff value is 1.095 for ${ }^{18}$ F-Alfatide II SUV max 0.81 for ${ }^{18}$ F-Alfatide II SUV ${ }_{\text {mean }}$ and 2.5 for ${ }^{18}$ F-Alfatide II T/NT. Although the AUCs $(<0.9)$ of ${ }^{18}$ F-Alfatide II are all less than those $(>0.9)$ of ${ }^{18} \mathrm{~F}-\mathrm{FDG},{ }^{18} \mathrm{~F}$-Alfatide II T/NT has the highest Youden index $(76.50 \%)$, specificity (100\%), accuracy $(89.19 \%)$ and PPV (100\%) among all these parameters. These data suggested that ${ }^{18} \mathrm{~F}$-Alfatide II T/NT may have the potential to serve as the most important semiquantitative parameter to be used for evaluation of ALN.

In the previous study, ${ }^{18} \mathrm{~F}$-Alfatide uptakes in malignant lymph nodes were significantly higher than those in benign lymph nodes for non-small cell lung cancer patients (22). Our results also showed that MALN had higher ${ }^{18} \mathrm{~F}$-Alfatide II uptake than BALN, as well as ${ }^{18} \mathrm{~F}$-FDG uptake. These data suggested that both ${ }^{18} \mathrm{~F}$-Alfatide II and ${ }^{18} \mathrm{~F}$-FDG are suitable to distinguish between MALN and BALN. However, no difference was found in ${ }^{18} \mathrm{~F}$-Alfatide II and ${ }^{18} \mathrm{~F}$-FDG uptakes among the primary breast tumors with MALN and with BALN. Several previous studies demonstrated that the higher ${ }^{18} \mathrm{~F}-\mathrm{FDG}$ uptake of the primary breast lesion could mean a higher incidence of ALN metastasis $(8,34-36)$. Other studies however revealed no 
significant difference between MALN and BALN in terms of primary breast mass in ${ }^{18} \mathrm{~F}-\mathrm{FDG}$ PET/CT (37, 38), which was also found in the present study for both ${ }^{18} \mathrm{~F}$-Alfatide II and ${ }^{18} \mathrm{~F}$-FDG. Song et al. thought molecular subtypes should be considered as a possible factor to affect ${ }^{18} \mathrm{~F}$-FDG uptake by primary breast cancer lesion for predicting ALN metastasis (8). Kim et al. demonstrated that ${ }^{18} \mathrm{~F}$-FDG uptake of primary breast cancer lesion has a significant predictive value for ALN metastasis in patients with ERpositive/HER-2-negative and HER-2-positive subtypes (39). More luminal B subtype and triple-negative subtype were included in our study, which could cause no significant difference in primary breast tumors with MALN versus BALN for ${ }^{18} \mathrm{~F}$-FDG and ${ }^{18} \mathrm{~F}$-Alfatide II.

There are some limitations in the present study. Particularly, the number of cases is not large enough. Due to the small number of luminal A and HER-2 overexpressing subtype, the evaluation of ALN using ${ }^{18} \mathrm{~F}-$ Alfatide II in each subtype was not conducted in this study. In the future, further investigations are required to concentrate on exploring the preference of ${ }^{18} \mathrm{~F}$-Alfatide II in assessing ALN for some certain subtypes of breast cancer.

\section{Conclusion}

${ }^{18} \mathrm{~F}$-Alfatide II can be used to detect metastatic ALN like ${ }^{18} \mathrm{~F}-\mathrm{FDG}$ with high specificity but relatively low sensitivity. Combining ${ }^{18} \mathrm{~F}$-Alfatide II and ${ }^{18} \mathrm{~F}$-FDG can significantly improve sensitivity and NPV. ${ }^{18} \mathrm{~F}$ Alfatide II is insufficient for assessing ALN of triple-negative subtype but complementary to ${ }^{18} \mathrm{~F}-\mathrm{FDG}$ for ALN of luminal B subtype. Having the highest specificity, accuracy and PPV, ${ }^{18}$ F-Alfatide II T/NT may be the most important semi-quantitative parameter for the evaluation of ALN. PET/CT can distinguish between MALN and BALN based on ${ }^{18} \mathrm{~F}$-Alfatide II uptake of the ALN, but can't do based on ${ }^{18} \mathrm{~F}$-Alfatide II uptake of the primary breast tumor.

\section{Declarations}

\section{Compliance with Ethical Standards:}

\section{Conflict of Interest:}

All authors declare no conflict of interest.

\section{Ethical approval:}

All procedures performed in studies involving human participants were in accordance with the ethical standards of the institutional and/or national research committee and with the 1964 Helsinki declaration and its later amendments or comparable ethical standards.

\section{Informed consent:}


Informed consent was obtained from all individual participants included in the study.

Funding: This study was funded by $\mathrm{X}$ (grant number $\mathrm{X}$ ).

This work was supported by the National Key Basic Research Program of China (973 program, 2014CB744504), the National Natural Science Foundation of China (81971675), the Natural Science Foundation of Jiangsu Province (BK20160610), Jiangsu Province Social Development Program (BE2017772), Jiangsu Planned Projects for Postdoctoral Research Funds (1601090C), and the National University of Singapore Start-up Fund (R-180-000-017-133, R-180-000-017-733, R-180-000-017-731).

\section{References}

1. Kvistad KA, Rydland J, Smethurst HB, et al. Axillary lymph node metastases in breast cancer: preoperative detection with dynamic contrast-enhanced MRI. Eur Radiol. 2000;10:1464-1471.

2. Jessing $C$, Langhans $L$, Jensen $M B$, et al. Axillary lymph node dissection in breast cancer patients after sentinel node biopsy. Acta Oncol. 2018;57:166-169.

3. Zhang X, Liu Y, Luo H, et al. PET/CT and MRI for identifying axillary lymph node metastases in breast cancer patients: systematic review and meta-analysis. J Magn Reson Imaging. 2020;52:1840-1851.

4. Chang JM, Leung JWT, Moy L, et al. Axillary nodal evaluation in breast cancer: state of the art. Radiology. 2020;295:500-515.

5. Zhao M, Wu Q, Guo L, et al. Magnetic resonance imaging features for predicting axillary lymph node metastasis in patients with breast cancer. Eur J Radiol. 2020;129:109093.

6. Zhou P, Wei Y, Chen G, et al. Axillary lymph node metastasis detection by magnetic resonance imaging in patients with breast cancer: a meta-analysis. Thorac Cancer. 2018;9:989-996.

7. Orsaria P, Chiaravalloti A, Caredda E, et al. Evaluation of the usefulness of FDG-PET/CT for nodal staging of breast cancer. Anticancer Res. 2018;38:6639-6652.

8. Song BI, Kim HW, Won KS. Predictive value of ${ }^{18} \mathrm{~F}-\mathrm{FDG}$ PET/CT for axillary lymph node metastasis in invasive ductal breast cancer. Ann Surg Oncol. 2017;24:2174-2181.

9. Leenders M, Kramer G, Belghazi K, et al. Can we identify or exclude extensive axillary nodal involvement in breast cancer patients preoperatively? J Oncol. 2019;2019:8404035.

10. Felding-Habermann B, O'Toole TE, Smith JW, et al. Integrin activation controls metastasis in human breast cancer. Proc Natl Acad Sci U S A. 2001;98:1853-1858.

11. Chen $\mathrm{H}, \mathrm{Niu} \mathrm{G}, \mathrm{Wu} \mathrm{H}$, et al. Clinical application of radiolabeled RGD peptides for PET imaging of integrin $a_{v} \beta_{3}$. Theranostics. 2016;6:78-92.

12. Yu C, Pan D, Mi B, et al. ${ }^{18} \mathrm{~F}$-Alfatide II PET/CT in healthy human volunteers and patients with brain metastases. Eur J Nucl Med Mol Imaging. 2015;42:2021-2028.

13. Mi B, Yu C, Pan D, et al. Pilot prospective evaluation of ${ }^{18} \mathrm{~F}$-Alfatide II for detection of skeletal metastases. Theranostics. 2015;5:1115-1121. 
14. Wan W, Guo N, Pan D, et al. First experience of ${ }^{18} \mathrm{~F}$-alfatide in lung cancer patients using a new lyophilized kit for rapid radiofluorination. J Nucl Med. 2013;54:691-698.

15. Luan X, Huang Y, Gao S, et al. ${ }^{18} \mathrm{~F}$-alfatide PET/CT may predict short-term outcome of concurrent chemoradiotherapy in patients with advanced non-small cell lung cancer. Eur J Nucl Med Mol Imaging. 2016;43:2336-2342.

16. Liu J, Wang D, Meng X, et al. ${ }^{18} \mathrm{~F}$-alfatide positron emission tomography may predict antiangiogenic responses. Oncol Rep. 2018;40:2896-2905.

17. Wu C, Yue $X$, Lang $L$, et al. Longitudinal PET imaging of muscular inflammation using ${ }^{18} \mathrm{~F}$-DPA-714 and ${ }^{18} \mathrm{~F}$-Alfatide II and differentiation with tumors. Theranostics. 2014;4:546-555.

18. Bao X, Wang MW, Luo JM, et al. Optimization of early response monitoring and prediction of cancer antiangiogenesis therapy via noninvasive PET molecular imaging strategies of multifactorial bioparameters. Theranostics. 2016;6:2084-2098.

19. Guo J, Guo N, Lang L, et al. ${ }^{18} \mathrm{~F}$-alfatide II and ${ }^{18} \mathrm{~F}$-FDG dual-tracer dynamic PET for parametric, early prediction of tumor response to therapy. J Nucl Med. 2014;55:154-160.

20. Dong Y, Wei Y, Chen G, et al. Relationship between clinicopathological characteristics and PET/CT uptake in esophageal squamous cell carcinoma: ${ }^{18} \mathrm{~F}$-Alfatide versus ${ }^{18} \mathrm{~F}$-FDG. Mol Imaging Biol. 2019;21:175-182.

21. Wu J, Wang S, Zhang X, et al. ${ }^{18} \mathrm{~F}$-Alfatide II PET/CT for identification of breast cancer: a preliminary clinical study. J Nucl Med. 2018;59:1809-1816.

22. Zhou Y, Gao S, Huang Y, et al. A pilot study of ${ }^{18} \mathrm{~F}$-Alfatide PET/CT imaging for detecting lymph node metastases in patients with non-small cell lung cancer. Sci Rep. 2017;7:2877.

23. Wahl RL, Siegel BA, Coleman RE, et al. Prospective multicenter study of axillary nodal staging by positron emission tomography in breast cancer: a report of the staging breast cancer with PET study group. J Clin Oncol. 2004;22:277-285.

24. Fuster $D$, Duch J, Paredes $P$, et al. Preoperative staging of large primary breast cancer with $\left[{ }^{18} \mathrm{~F}\right]$ fluorodeoxyglucose positron emission tomography/computed tomography compared with conventional imaging procedures. J Clin Oncol. 2008;26:4746-4751.

25. Heusner TA, Kuemmel S, Hahn S, et al. Diagnostic value of full-dose FDG PET/CT for axillary lymph node staging in breast cancer patients. Eur J Nucl Med Mol Imaging. 2009;36:1543-1550.

26. Groheux D, Giacchetti S, Moretti JL, et al. Correlation of high ${ }^{18} \mathrm{~F}-\mathrm{FDG}$ uptake to clinical, pathological and biological prognostic factors in breast cancer. Eur J Nucl Med Mol Imaging. 2011;38:426-435.

27. Robertson IJ, Hand F, Kell MR. FDG-PET/CT in the staging of local/regional metastases in breast cancer. Breast. 2011;20:491-494.

28. Pritchard KI, Julian JA, Holloway CM, et al. Prospective study of $2-\left[{ }^{18} \mathrm{~F}\right]$ fluorodeoxyglucose positron emission tomography in the assessment of regional nodal spread of disease in patients with breast cancer: an Ontario clinical oncology group study. J Clin Oncol. 2012;30:1274-1279. 
29. Kitajima K, Fukushima K, Miyoshi Y, et al. Diagnostic and prognostic value of ${ }^{18}$ F-FDG PET/CT for axillary lymph node staging in patients with breast cancer. Jpn J Radiol. 2016;34:220-228.

30. Sasada S, Masumoto N, Kimura Y, et al. Identification of axillary lymph node metastasis in patients with breast cancer using dual-phase FDG PET/CT. AJR Am J Roentgenol. 2019;213:1129-1135.

31. Zhang $X$, Liu Y, Luo H, et al. PET/CT and MRI for identifying axillary lymph node metastases in breast cancer patients: systematic review and meta-analysis. J Magn Reson Imaging. 2020;52:1840-1851.

32. Osborne JR, Port E, Gonen M, et al. ${ }^{18} \mathrm{~F}$-FDG PET of locally invasive breast cancer and association of estrogen receptor status with standardized uptake value: microarray and immunohistochemical analysis. J Nucl Med. 2010;51:543-550.

33. Park HL, Yoo IR, O JH, et al. Clinical utility of ${ }^{18} \mathrm{~F}-\mathrm{FDG}$ PET/CT in low ${ }^{18} \mathrm{~F}$-FDG-avidity breast cancer subtypes: comparison with breast US and MRI. Nucl Med Commun. 2018;39:35-43.

34. Seok JW, Kim Y, An YS, et al. The clinical value of tumor FDG uptake for predicting axillary lymph node metastasis in breast cancer with clinically negative axillary lymph nodes. Ann Nucl Med. 2013;27:546-553.

35. Yoo J, Kim BS, Yoon HJ. Predictive value of primary tumor parameters using ${ }^{18} \mathrm{~F}-\mathrm{FDG}$ PET/CT for occult lymph node metastasis in breast cancer with clinically negative axillary lymph node. Ann Nucl Med. 2018;32:642-648.

36. Jung NY, Kim SH, Kang BJ, et al. The value of primary tumor ${ }^{18} \mathrm{~F}-\mathrm{FDG}$ uptake on preoperative PET/CT for predicting intratumoral lymphatic invasion and axillary nodal metastasis. Breast Cancer. 2016;23:712-717.

37. Can C, Komek H. Metabolic and volume-based parameters of ${ }^{18} \mathrm{~F}-\mathrm{FDG}$ PET/CT for primary mass and axillary lymph node metastasis in patients with invasive ductal carcinoma: a retrospective analysis in relation to molecular subtype, axillary lymph node metastasis and immunohistochemistry and inflammatory markers. Nucl Med Commun. 2019;40:1051-1059.

38. Arslan E, Cermik TF, Trabulus FDC, et al. Role of ${ }^{18}$ F-FDG PET/CT in evaluating molecular subtypes and clinicopathological features of primary breast cancer. Nucl Med Commun. 2018;39:680-690.

39. Kim JY, Lee SH, Kim S, et al. Tumour ${ }^{18}$ F-FDG uptake on preoperative PET/CT may predict axillary lymph node metastasis in ER-positive/HER2-negative and HER2-positive breast cancer subtypes. Eur Radiol. 2015;25:1172-1181.

\section{Figures}


${ }^{18}$ F-Alfatide II

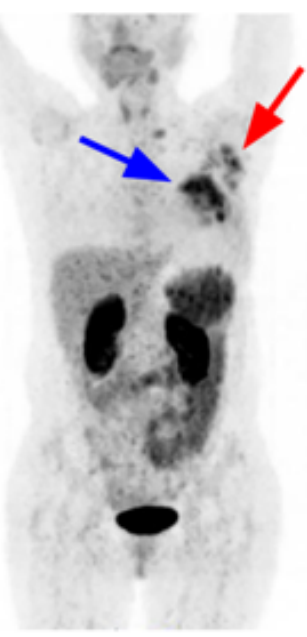

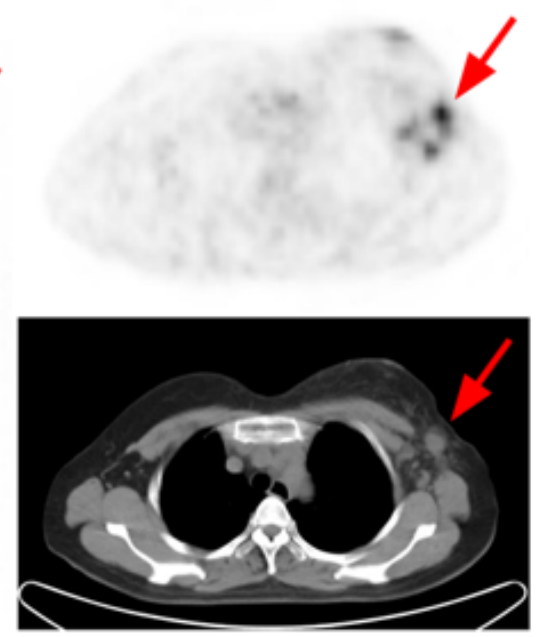

${ }^{18}$ F-FDG
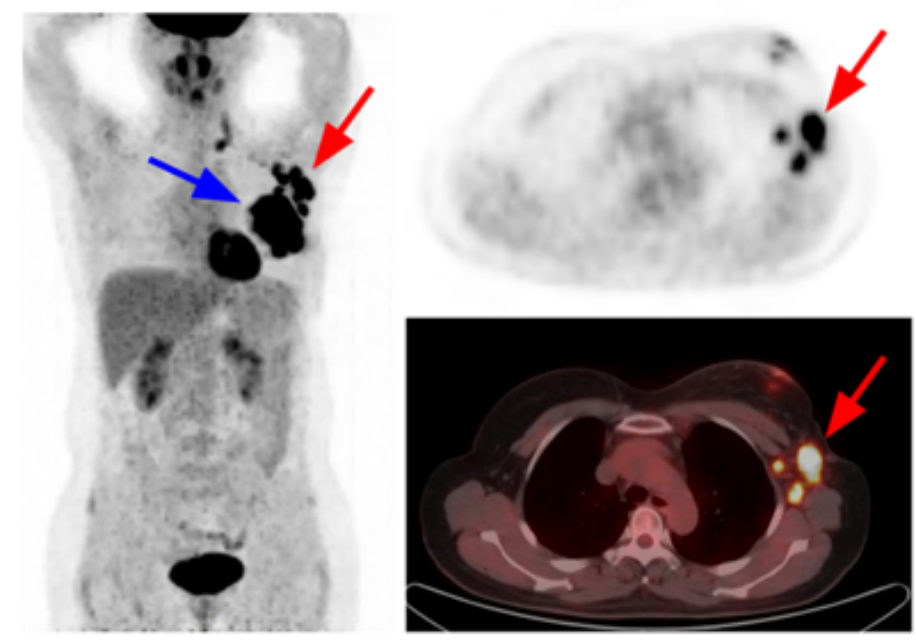

Figure 1

A 49-y-old patient with HER-2-overexpressing breast cancer (blue arrows) and ALN metastases (red arrows) showing high uptakes of both 18F-Alfatide II and 18F-FDG.

\section{${ }^{18}$ F-Alfatide II}

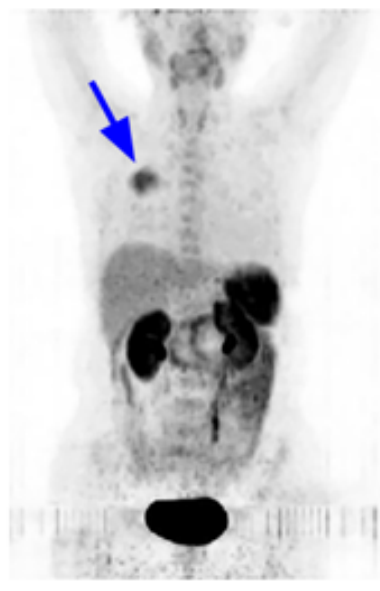

${ }^{18}$ F-FDG

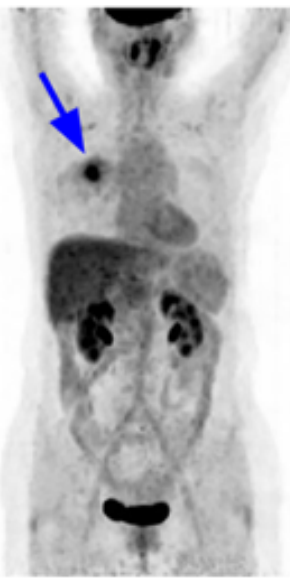

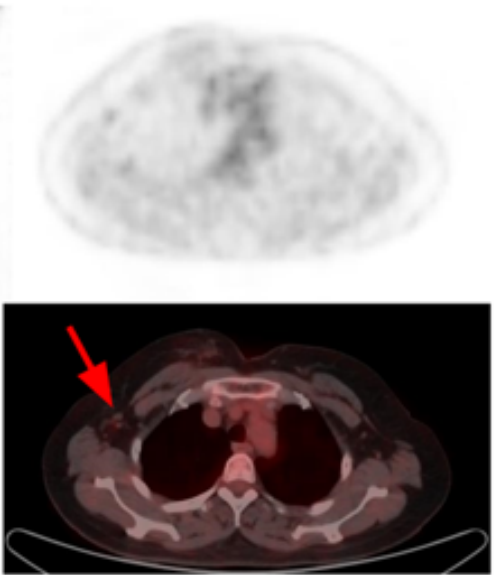

Figure 2

A 47-y-old patient with luminal B breast cancer (blue arrows) showing intense uptake of both 18F-Alfatide II and 18F-FDG, but no uptake of neither tracer in metastatic ALN (red arrows). 
${ }^{18}$ F-Alfatide II

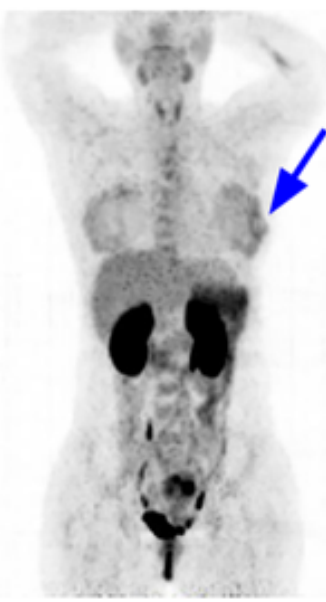

${ }^{18}$ F-FDG

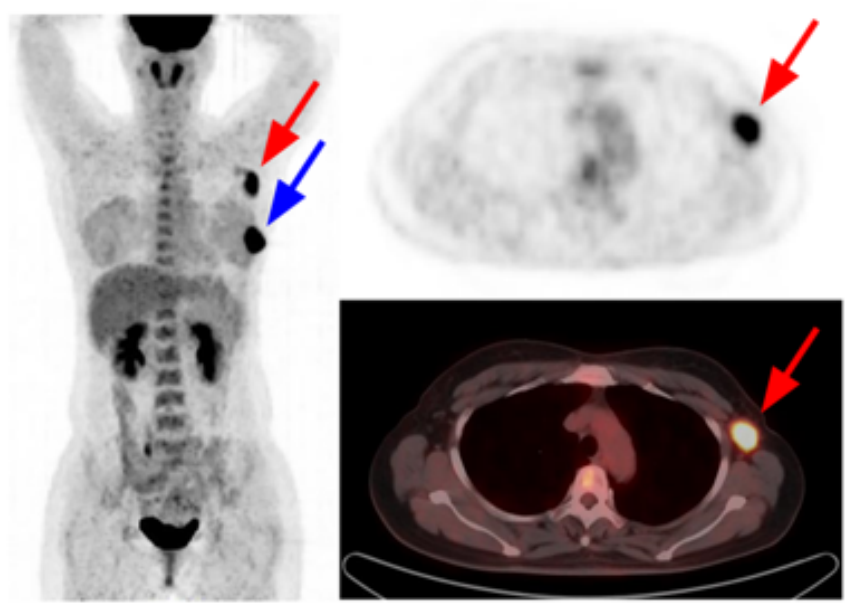

Figure 3

A 46-y-old patient with triple-negative breast cancer (blue arrows) and ALN metastasis (red arrows) showing no increased 18F-Alfatide II uptake but high 18F-FDG uptake.

\section{${ }^{18} \mathrm{~F}$-Alfatide II}

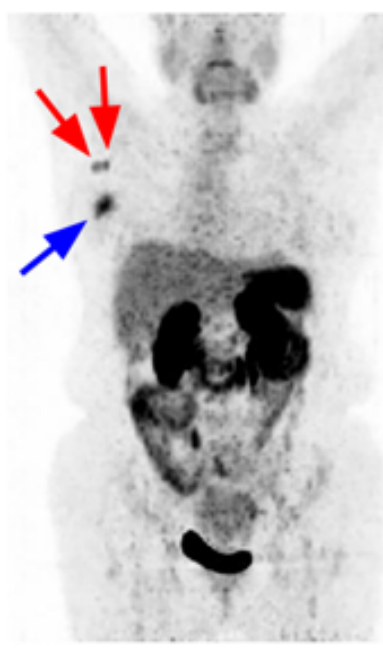

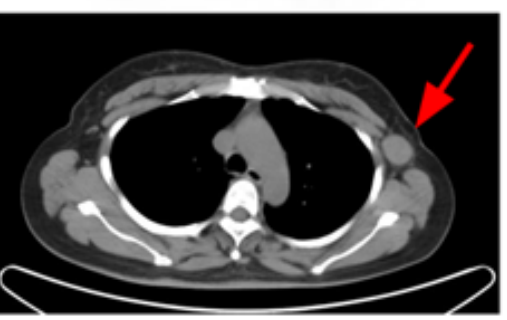
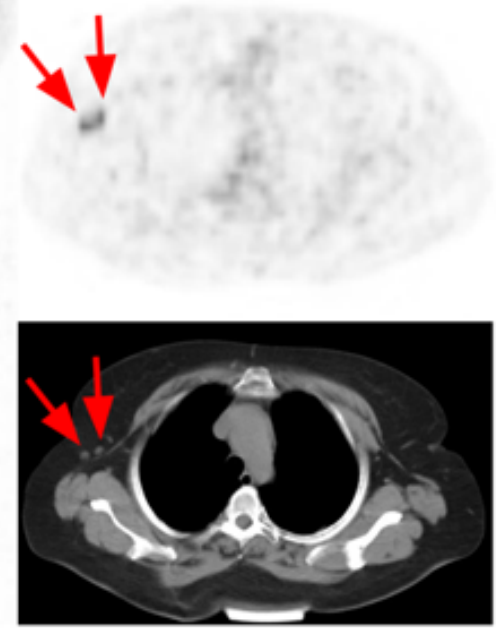

${ }^{18}$ F-FDG
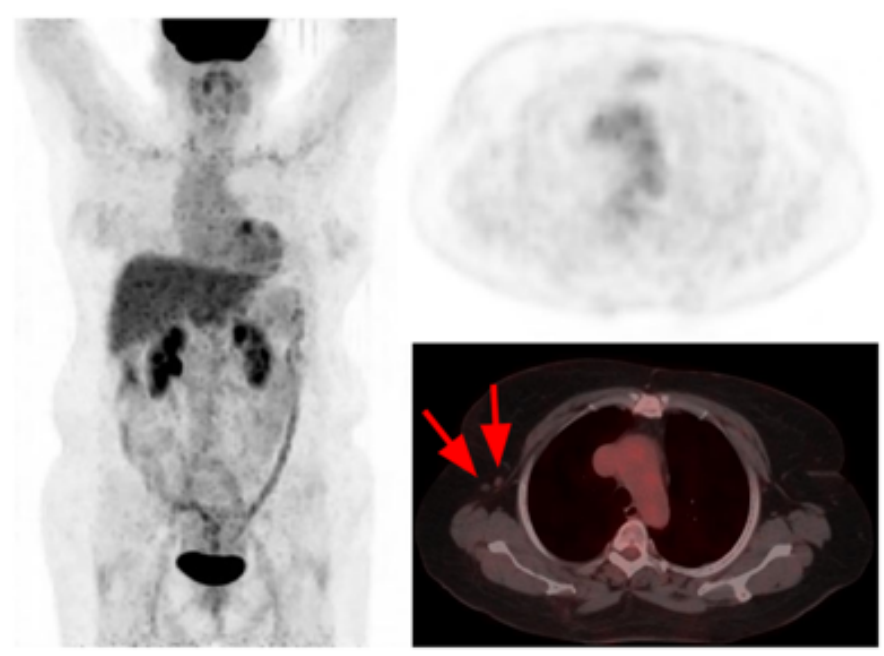

\section{Figure 4}

A 60-y-old patient with luminal B breast cancer (blue arrow) and ALN metastasis (red arrows) showing intense 18F-Alfatide II uptake but no 18F-FDG uptake in neither the primary tumor nor metastatic ALN. 


\section{${ }^{18}$ F-Alfatide II}

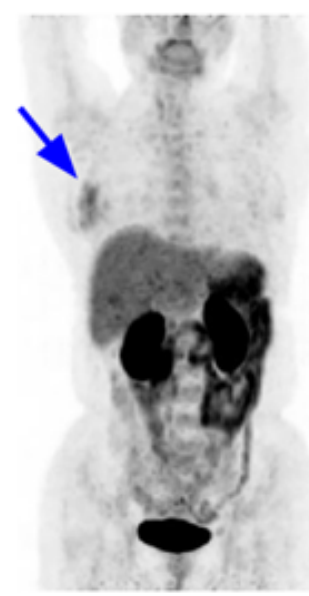

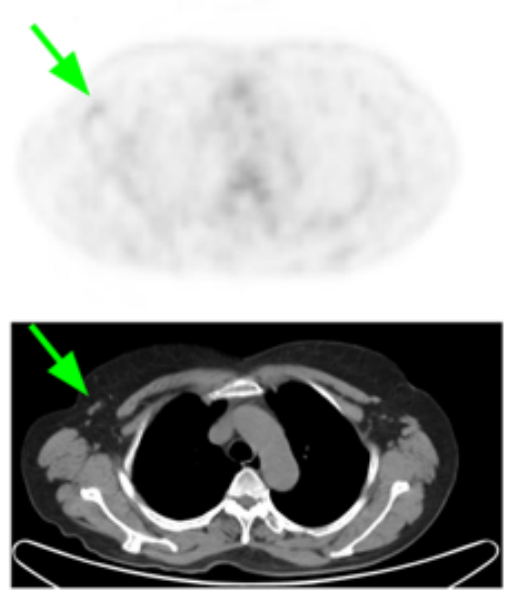
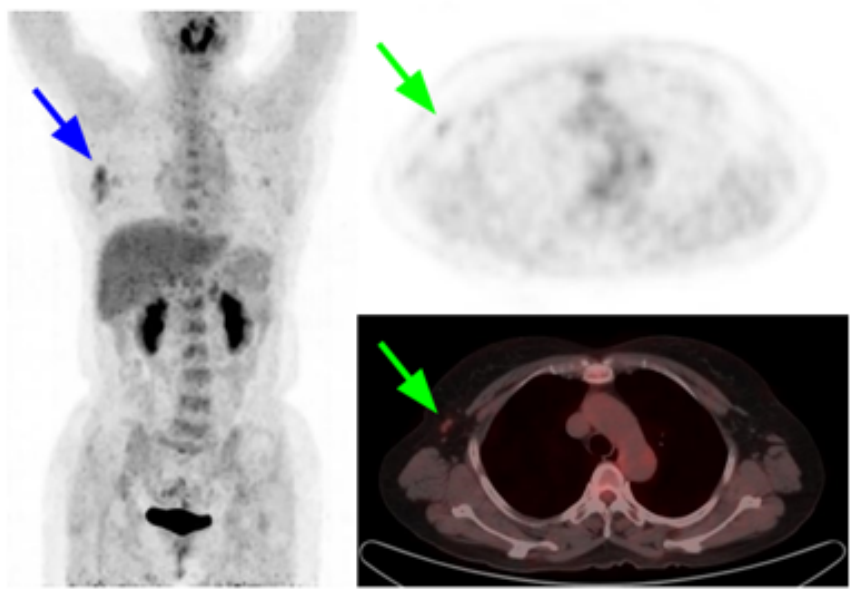

Figure 5

A 57-y-old patient with ductal carcinoma in situ (blue arrows) showed intense uptakes of both 18FAlfatide II and 18F-FDG, and non-metastatic ALN (green arrows) had slightly increased uptakes of both 18F-Alfatide II and 18F-FDG.

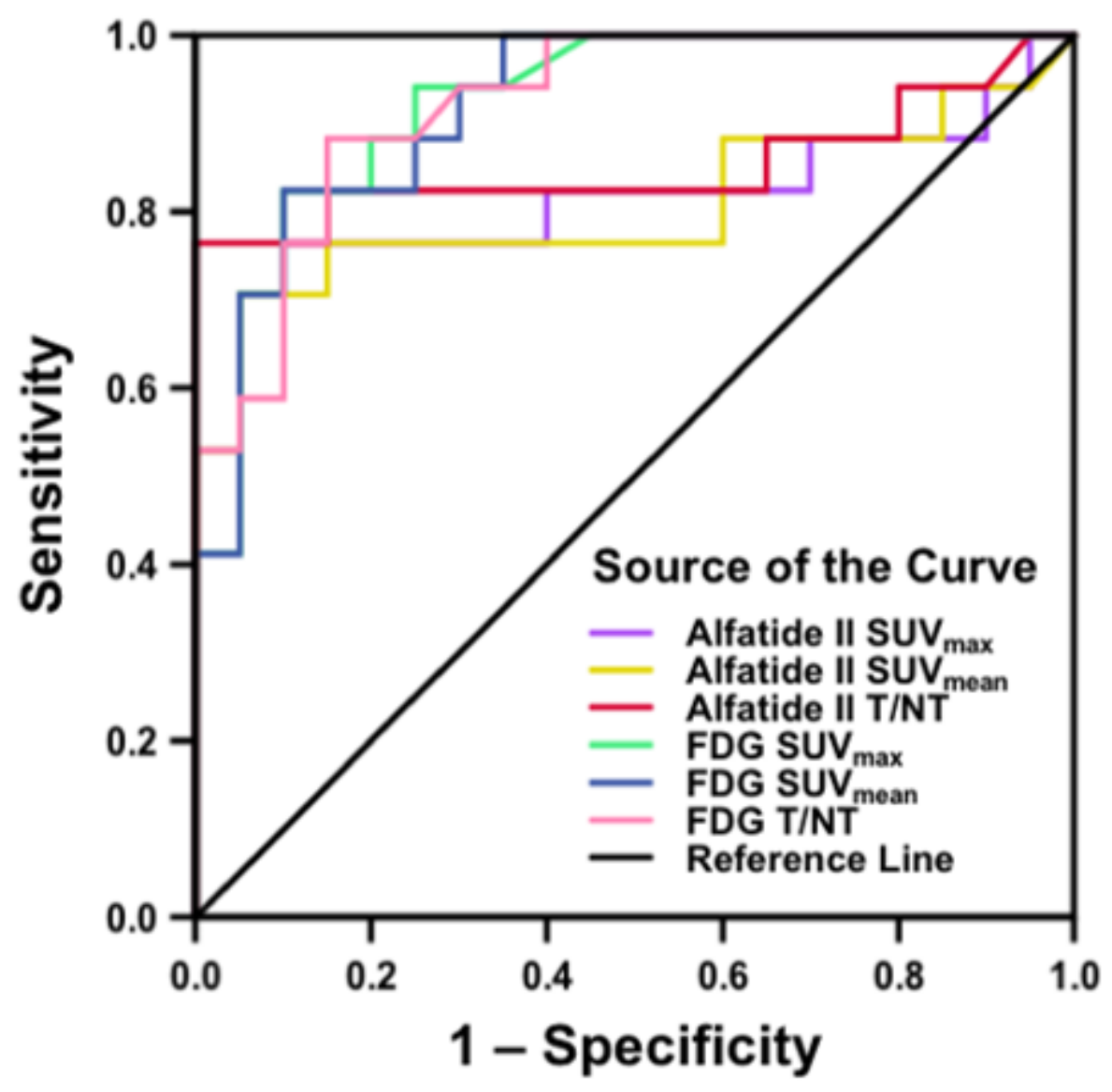

Figure 6 
ROC curves of 18F-Alfatide II and 18F-FDG parameters in identifying ALN metastasis. 